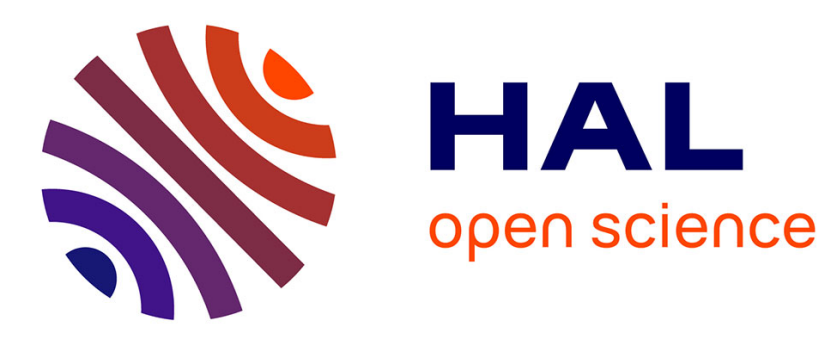

\title{
RVC-CAL dataflow implementations of MPEG AVC/H.264 CABAC decoding
}

Endri Bezati, Marco Mattavelli, Mickael Raulet

\section{To cite this version:}

Endri Bezati, Marco Mattavelli, Mickael Raulet. RVC-CAL dataflow implementations of MPEG AVC/H.264 CABAC decoding. Design and Architectures for Signal and Image Processing (DASIP), 2010 Conference on, 2010, United Kingdom. pp.207 -213, 10.1109/DASIP.2010.5706266 . hal00565297

\section{HAL Id: hal-00565297 \\ https://hal.science/hal-00565297}

Submitted on 11 Feb 2011

HAL is a multi-disciplinary open access archive for the deposit and dissemination of scientific research documents, whether they are published or not. The documents may come from teaching and research institutions in France or abroad, or from public or private research centers.
L'archive ouverte pluridisciplinaire HAL, est destinée au dépôt et à la diffusion de documents scientifiques de niveau recherche, publiés ou non, émanant des établissements d'enseignement et de recherche français ou étrangers, des laboratoires publics ou privés. 


\title{
RVC-CAL dataflow implementations of MPEG AVC/H.264 CABAC decoding
}

\author{
Endri Bezati ${ }^{1}$, Marco Mattavelli ${ }^{1}$, Mickaël Raulet ${ }^{2}$ \\ Swiss Federal Institute of Technology, STI-SCI-MM ${ }^{1}$ \\ Institut National des Sciences Appliques de Rennes, IETR ${ }^{2}$ \\ endri.bezati@epfl.ch,marco.mattavelli@epfl.ch, mickael.raulet@insa-rennes.fr
}

\begin{abstract}
This paper presents the implementation of the CABAC entropy decoder in the RVC-CAL dataflow programming language. CABAC is the Context based Adaptive Binary Arithmetic Coding entropy decoder used in the AVC/H.264 main and high profile video standard. CABAC as an entropy decoding engine gives a better compression rate efficiency, but has a greater complexity compared to other entropy decoding engines. With the implementation of the CABAC entropy decoder in RVC-CAL, this paper is a proof that complex algorithms can be implemented in a high level design and that can be described in a data flow language as $R V C$ CAL is. Thought in this paper we are going to analyze two different methods of implementing the CABAC entropy decoder and propose a data flow model of the CABAC entropy decoder in RVC-CAL.
\end{abstract}

\section{Introduction}

\subsection{RVC Standard}

The purpose of the MPEG RVC standard is to offer a more flexible use and faster path to innovation of MPEG standards in a way that is competitive in the current dynamic environment. This is meant to give MPEG standards an edge over its market competitors by substantially reducing the Time to Market (TTM). The RVC initiative exploits the reuse of obvious commonalities among different MPEG standards and their possible extensions using appropriate higher level formalisms. Thus the objective of the RVC standard is to describe current and future codecs in a way that makes such commonalities explicit, reducing the implementation burden for device vendors. In order to achieve this objective, RVC suggests simplifying the specification of new coding tools by reusing components of previous standards instead of defining new ones. [1,5]

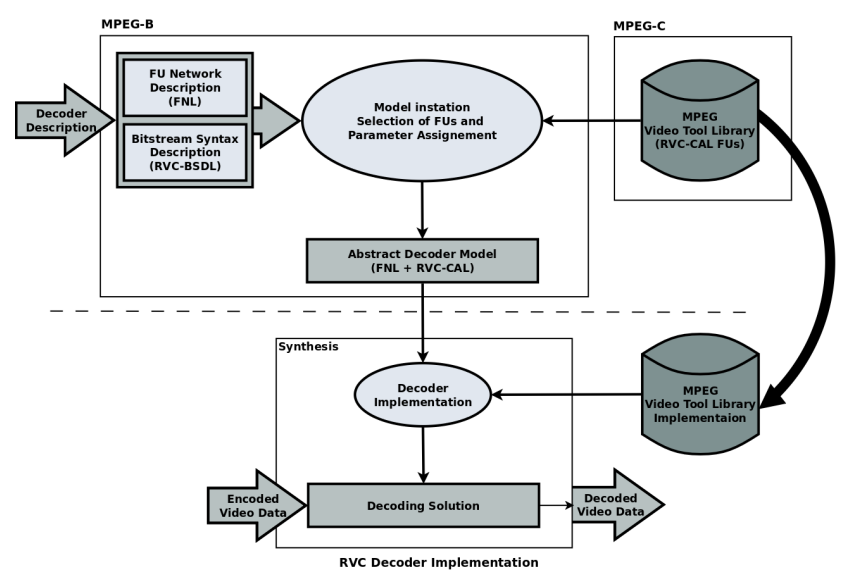

Figure 1. RVC Framework

The MPEG-B standard defines the MPEG RVC framework and the RVC-CAL dataflow programming language. Also in the MPEG RVC framework the MPEG-C standard defines the library of the video coding tools (Video Tool Library or VTL). The Figure 1 describes how from an abstract decoder model using a Functional Unit (FU) network description a decoding solution can be achieved for a hardware or software implementation.

\subsection{CAL Language}

CAL Actor Language is a language based on the Actor model of computation for data flow systems. It provides many natural concepts to facilitate modeling of those systems. An actor is a modular component that encapsulates its own state. Each actor interacts with each other through FIFO channels, see Figure 2. An actor in general contains global variables, state variables, global parameters, actions, procedures, functions and finite state machine to control the executions of actions. CAL enables concurrent development and has a strong encapsulation. CAL is used in a variety of applications and has been compiled to hardware and software implementations. The RVC-CAL language is a subset 
of the CAL language the has been normalized by ISO/IEC as a part of the RVC standard. Although it has some restrictions in data types and feature that are in used in $\mathrm{CAL}[1,3]$.

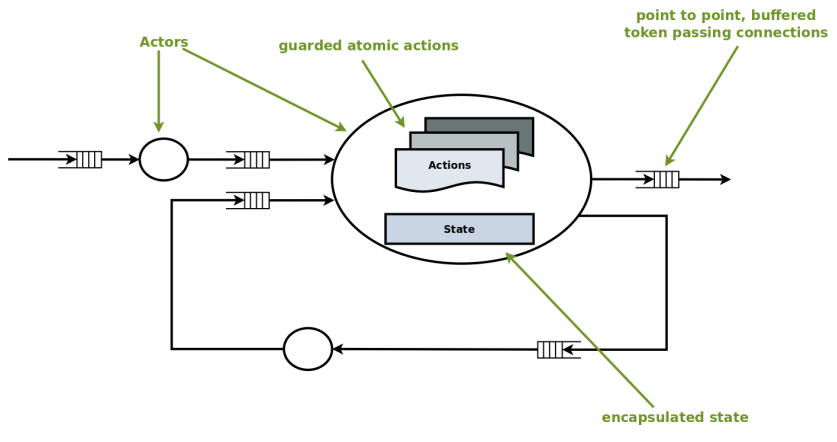

Figure 2. The CAL computing model

\subsection{Open RVC-CAL Compiler}

The Open RVC-CAL Compiler is a tool set which provides developers with a compiler infrastructure to generated source code in several languages from the RVC-CAL actors and XDF networks (a network of actors) [8]

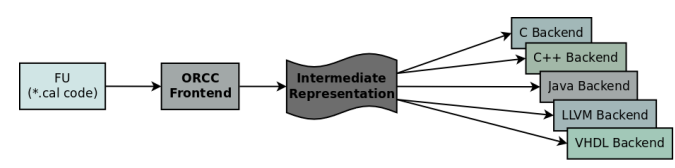

Figure 3. ORCC Framework

- Functional Unit (FU): is a video processing component or an actor in CAL language. The RVC framework provides a Video Tool Library which is consisted by a set of FUs that can be embedded by the client and combined for building the required decoder

- Frontend \& IR : For a RVC-CAL dataflow program to be compiled, its compilation process its done in twosteps process. The fronted will parse all actors and it will translates them to an Intermediate Representation (IR) which is serialized to one file per actor in a JSONbased format (an XML file format). The IR is a data structure that is constructed from input data (the actor) to a program, and from which part or all of the output data of the program is constructed in turn. The next steps is to run a language target-specific back-end.

- Backends: Depending the target, ORCC offers a variety of back-ends. Their purpose is to create target specific code. Each backend will parse the hierarchical network from a top-level network and its child network. Also optionally it flattens the hierarchical net- work. ORCC for the moment offers a variety of backends. These back-ends are C, C++, LLVM, VHDL and a partial support for the Xlim code generation.

To generate a software decoding solution we used the $\mathrm{C}$ backend of ORCC. The generated C code is ANSI-C compatible and it is portable to different platforms such as Windows, Linux, Mac OS X and others.

\subsection{Entropy Encoding}

In information theory an entropy encoding is a lossless data compression scheme that is independent of the specific characteristics of the medium. In the AVC/H.264 three types of entropy encoding are used. Exp-Golomb for decoding the headers of the bitstream(video file). Furthermore the description of a macroblock and its quantized coefficients are encoded using the CAVLC or CABAC encoding.

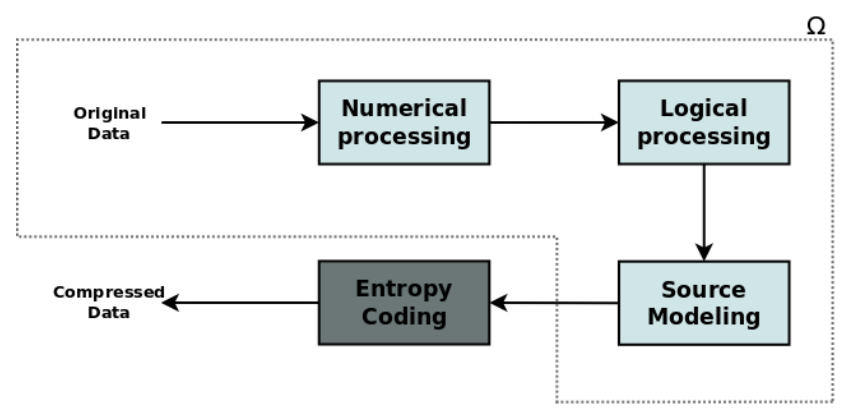

Figure 4. Entropy encoding as the final stage of compression

\section{CABAC Entropy Decoder}

CABAC or Context Adaptive Binary Arithmetic Coding is an entropy decoder that gives better compression rates than the Baseline Profile CAVLC (Context Adaptive Variable Length Coding) entropy decoder. CABAC chooses a probability model for each syntax element, it adopts the estimated probability based on local statistics and it uses an arithmetic coder rather than variable-length coding (CAVLC). Though CAVLC is a lot more easy to implement and less power hungry, CAVLC gets the value directly by reading the bit-stream. However with CABAC the complexity is more important as there is a feedback loop between the context modeler and the arithmetic coder [2].

The H.264/AVC parser depending the context, demands the entropy decoding engine for the value of an Syntax Element (SE), see Figure 5. An SE is an element of data represented in the bit-stream, for example the Macro-block type Intra $4 \times 4$, Intra $16 \times 16$ or other. 


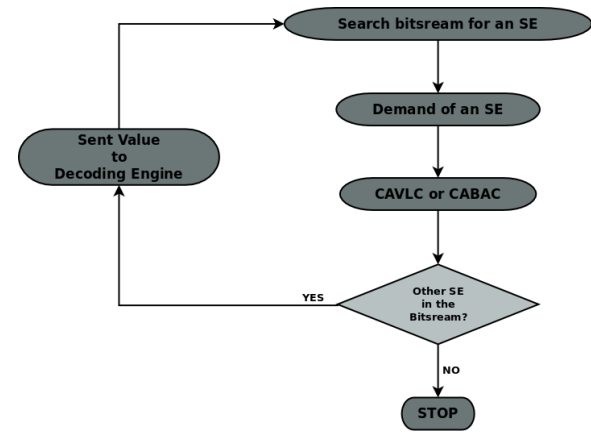

Figure 5. An simple interpretation of the H.264/AVC parser.

\subsection{Binarization Process}

Each SE has a particular binarization. In H.264/AVC four types of binarization are defined.

- Unary binarization, used for reference picture list reordering SE

- Truncate unary binarization, used for the Intra chroma prediction mode SE

- Concatenated unary/k-th order Exp-Golomb(UEKg) binarization, used mainly in the Residual block process and in the movement prediction for $\mathrm{P}$ and $\mathrm{B}$ slices

- Fixed Length binarization, used for flag types SE

An example of the Fixed Length binarization process can be seen in the table below. The binIdx, indicates the binary index of the binary string. For example this same binary strings indicates the values of the rem_intra_4x4_pred_mode and rem_intra_8x8_pred_mode SE from the H.264/AVC standard.

\begin{tabular}{|c|c|}
\hline Value of Syntax Element & Bin String \\
\hline 0 & 000 \\
\hline 1 & 100 \\
\hline 2 & 010 \\
\hline 3 & 110 \\
\hline 4 & 001 \\
\hline 5 & 101 \\
\hline 6 & 011 \\
\hline 7 & 111 \\
\hline binIdx & 012 \\
\hline
\end{tabular}

Table 1. FL binarization with $\mathrm{cMax}=7$.

\subsection{CABAC Variables}

In the H.264/AVC standard the prediction of the binary value is basically calculated by these variables.

- ctxIdx, which indicates the context of the syntax element

- codIRange and codIOffset, corresponds to the status of the arithmetic decoding process

- $p$ StateIdx, corresponds to the probability state index

- valMPS, corresponds to the value of the most probable symbol

\subsection{CABAC Arithmetic coding engine}

After the binarization of an SE, each bin in the binarized string will be given an context (ctxIdx) and then encoded using the CABAC arithmetic coding. The Context Arithmetic coding theory is based on the principle of recursive interval subdivision. Given a probability estimation $p_{0}$ and $p_{1}=1-p_{0}$ of the binary decision $(0,1)$, codIRange will be initially gives the code sub-interval, this range will be subdivided into two sub-intervals having the following range $p_{0} * \operatorname{codIR}$ Range and codIRange $-p_{0} * \operatorname{codI}$ Range, respectively. Depending on the decision, the corresponding sub-interval is going to be chosen as the new code interval, and the binary code string pointing into that interval will represent the sequence of observed binary decisions. In the decoding decision procedure it is useful to distinguish between the most probable symbol (MPS) and the least probable symbol (LPS), it is better that the binary decisions have to be identified as either MPS or LPS, rather than 0 or 1 . Given this terminology, each context is specified by the probability $p$ of the LPS and the value of MPS (valMPS), which is either 0 or 1. [7]

\section{RVC-CAL CABAC Implementation}

The implementation of the RVC H.264/AVC parser FU in the current RVC-CAL AVC CBP (Constrained Base Profile) [4], [6] decoder is being used as a base for the CABAC entropy decoder. That means that each action used by the parser from the CAVLC is modified so that can support CABAC SE's extraction. The CABAC implementation is modelized by twelve untagged actions. These actions are not named in the source code because they are executed outside the FSM (Finite State Machine) of the parser FU. Here we give them names for clarity. We developed two methods to retrieve the Syntax Elements values from the AVC/H.264 parser Functional Unit. The first method is using a Look Up Table (LUT) for comparing the decode binary string given 
by the CABAC procedure and the binarized Value of an Syntax Element. This method was later abandoned due to the complexity of the binarization LUT of the quantized coefficients (Macroblock residual values) and its slow double loop algorithm. Given the difficulty of the binarized residual values, we developed a new method, Bin By Bin, which is an adaptive decoding solution for each Syntax Element.

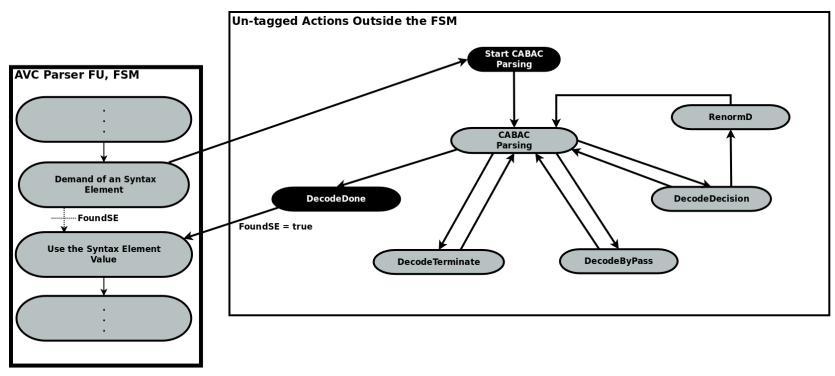

Figure 6. RVC-CAL main CABAC parsing actions

In the Figure 6 we represent the LUT and Bin by Bin method as CabacPasrsing, depending the method we want to use. CabacPasrsing is the main action and it controls the activation of the CABAC decoding actions. Depending the SE three actions are used to decode the binary value. These actions are DecodeDecision, DecodeByPass and DecodeTerminate. All three of them execute smaller actions to read the bitstream.

\subsection{Look Up Table method}

After the CABAC arithmetic decoding the decoded bins have to be de-binarized so the value can we retrieved. A very simple way to get the decoding value from CABAC de-binarization is to use a Look Up Table. As Each SE has a specific binarization the idea was to create a look up-table for each SE and to stock the binarized values in a multi dimension table. Thus index of the table will be the decoded value and the rows of the table represents binarized bin string. With this method bits are recovered one by one and then are compared with the binarization table of the searched SE. As we mentioned before we abandoned this method, but all the SE expect the coeff_abs_level_minus1 (quantized coefficients), ref_lx and mvd_lx (mouvement vectors) fully functional.

\subsection{Bin by Bin method}

Using a LUT demands more memory and the compare algorithm could be much more time consuming. For example to decode the 255 residual value of the coeff_abs_level_minus1 SE with LUT method, the algorithm

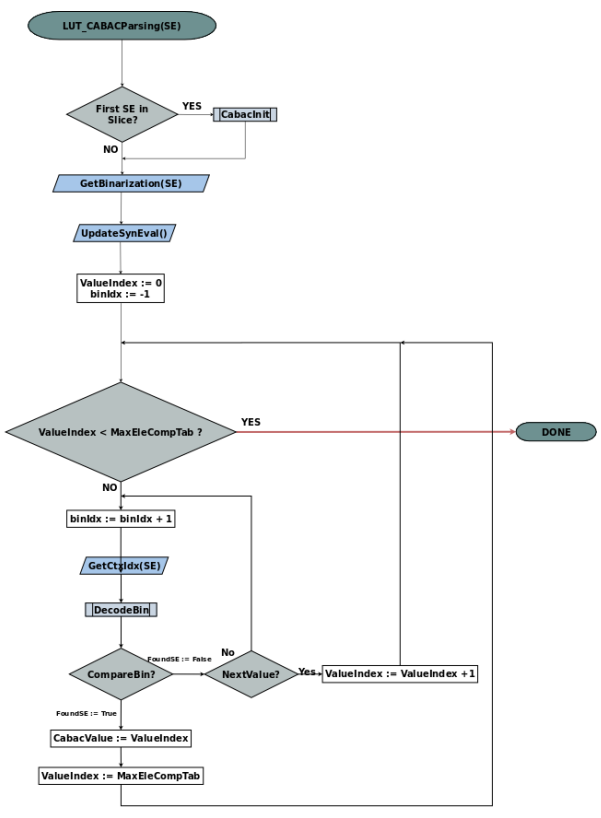

Figure 7. The LUT Method

needed to execute $255^{*}(\mathrm{n}$ bins) double loops. So to make the process more faster we created the Bin By Bin method. In this method each SE has a different de-binarization procedure. In this way the CABAC decoding is unique for each SE.
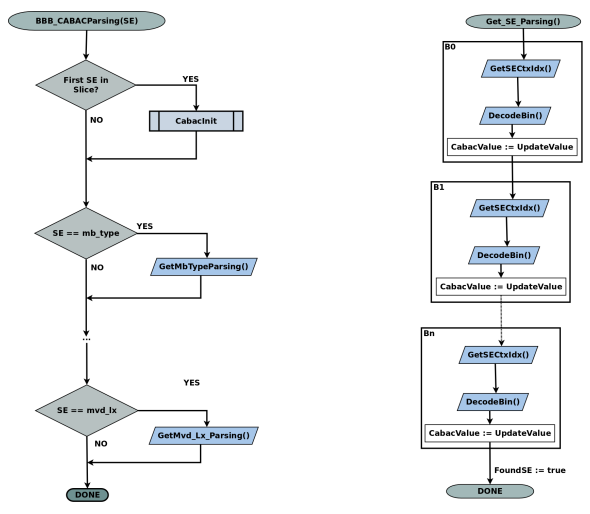

Figure 8. The Bin By Bin Method

The Figure 9 shows a part of the coeff_abs_level_minus 1 SE de-binarization process, this procedure is called as many times as the SE value is completely de-binarized.

\section{Results}

\subsection{Conformance Sequences support}

The RVC-CAL CABAC implementation supports all the Syntax Element found in the AVC/H.264 standard except 


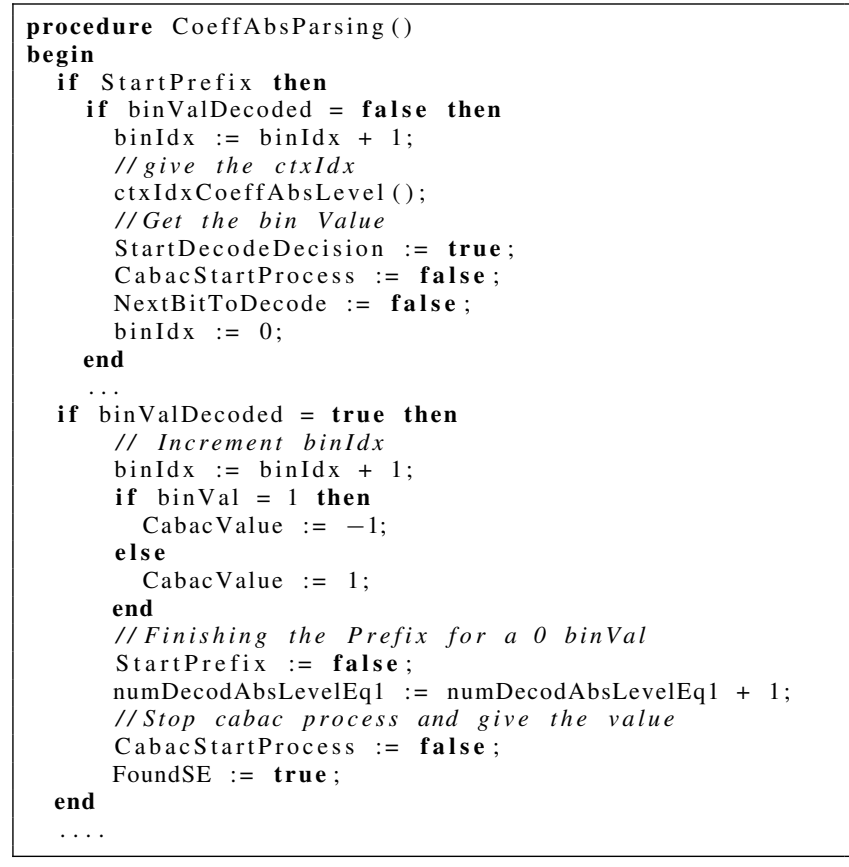

\section{Figure 9. A part of the coeff_abs_level_minus1 SE de-binarization procedure.}

the mb_field_decoding_flag (which indicates if the macroblock is field coded). Also because the abstract decoder model used in this implementation is coming for the AVC CBP FU, it does not support the decoding of the BiPred slices. Thus our CABAC implementation supports all the encoded Macroblock SE except those coded in field order. The following Table 2 is a list of supported conformance $\mathrm{AVC} / \mathrm{H} .264$ sequences coded using the CABAC entropy encoding. Because of the AVC CBP FU the sequence marked as $I P B$ are supported only by the AVC Parser FU, the decoding of those sequence is a work in progress of the AVC FRExt FU(Fidelity Range Extensions).

\subsection{RVC-CAL CABAC versus JM CABAC implementation}

The RVC-CAL is by design ment to be a high level language. The Decode Decision process is used by almost all Syntax Element in the H.264/AVC standard. Here we compare the interpretation of the H.264/AVC Decode Decision flowchart(Figure 10) with the RVC-CAL (Figure 11) and the $\mathrm{C}$ language used in the JM software Reference software of the H.264/AVC Standard (Figure 12).

In term of source code we have exactly the same line of codes for this two different implementations but the difference is in the readability and the understanding of the source code. The RVC-CAL implementation is an identi-

\begin{tabular}{|l|c|c|c|}
\hline Name & Slice Type & N. Frames & Status \\
\hline CABA1_Sony_D & I & 50 & Passed \\
CABA2_Sony_E & IP & 300 & Passed \\
CABA3_Sony_C & IPB & 300 & Only Parser \\
CABA3_TOSHIBA_E & IP & 300 & Passed \\
CABA1_SVA_B & I & 17 & Passed \\
CABA2_SVA_B & IP & 17 & Passed \\
CABA3_SVA_C & IPB & 33 & Only Parser \\
CANL1_TOSHIBA_G & I & 300 & Passed \\
CANL1_Sony_E & I & 17 & Passed \\
CANL2_Sony_E & IP & 300 & Passed \\
CANL3_SVA_C & IPB & 300 & Only Parser \\
CANL1_SVA_B & I & 17 & Passed \\
CANL2_SVA_B & I & 17 & Passed \\
CANL3_SVA_B & IP & 17 & Passed \\
CANL4_SVA_C & IPB & 33 & Only Parser \\
CAQP1_Sony_B & IP & 50 & Passed \\
CACQP3_Sony_D & IPB & 50 & Only Parser \\
\hline
\end{tabular}

Table 2. Conformance sequences supported by the Bin By Bin RVC-CAL implementation

cally copy the of the Decode Decision flowchart. In addition the $\mathrm{C}$ code of the JM software contains pointers, the variables are not named exactly the same and in general the code readability is not as easy to read and analysable as the RVC-CAL code.

Our full implementation of the CABAC in RVC-CAL contains less source code than the $\mathrm{C}$ implementation of the JM AVC decoder. 2500 source code lines for the RVC-CAL and more than 3000 lines for the $\mathrm{C}$ code.

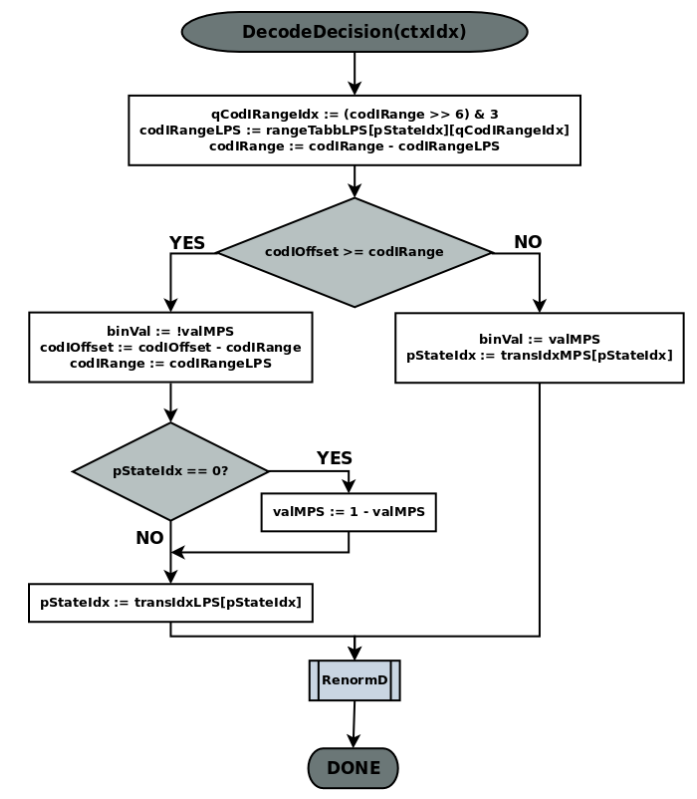

Figure 10. Decoding Decision flowchart in the H.264/AVC standard. 


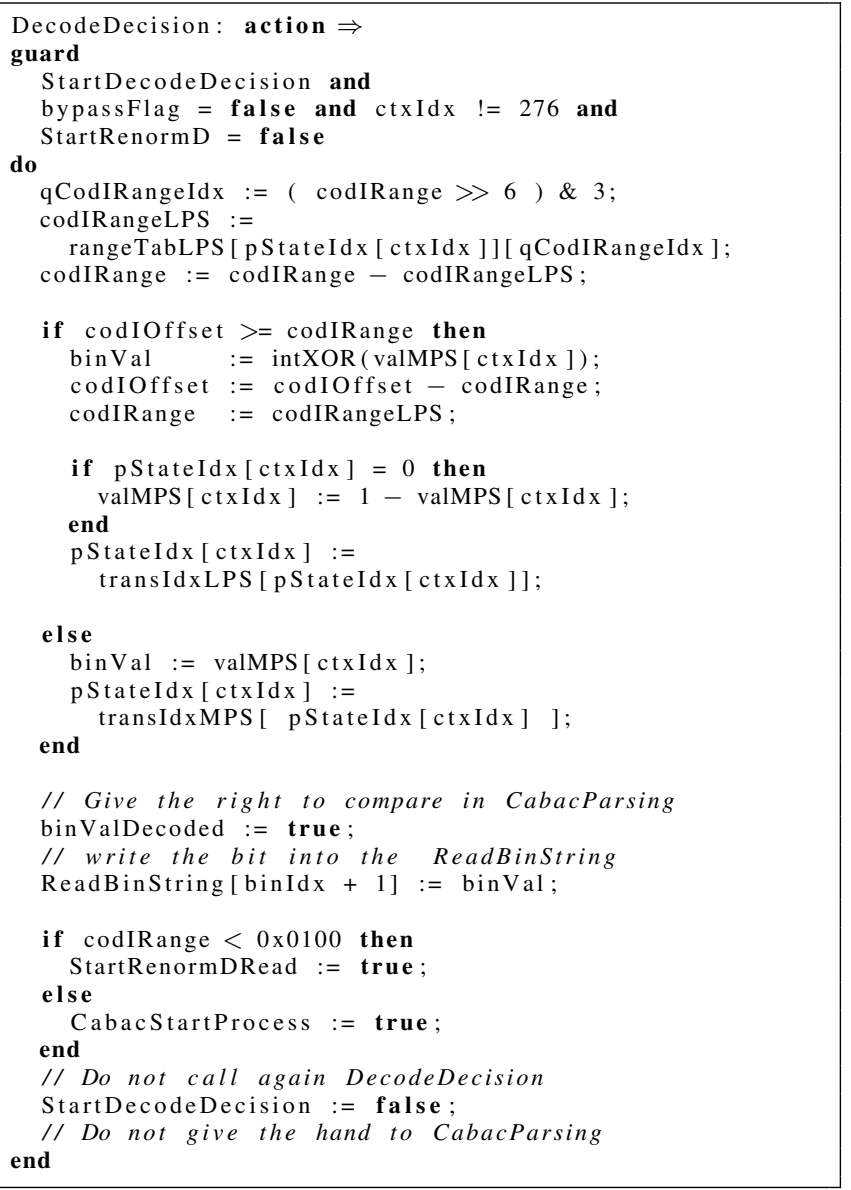

Figure 11. Decoding Decision action written in RVC-CAL.

\section{To a data flow model}

The actual form of the AVC parser in RVC-CAL is sequential, as is supposed to be for a parser. As RVC-CAL is data flow based language, the idea here is to transform it's sequential form to a data flow one. Actually the challenge is how to pipeline a sequential parser. As a matter of fact the bitsream has a start up code when a new slice starts. We can store the whole bitstream for a slice to buffer and then analyzed it and dispatched it to where is necessary. Actually in this way we can separate the parser process in smaller actors and create a pipeline architecture. These actors are the BitstreamAnalyser, NAL, SPS, PPS, SliceHeader and EntropyDecoding. Appart EntropyDecoding all other actors are explicit, for a reconfigurable decoder is a necessity to separate the CAVLC and the CABAC entropy Decoding. So in the EntropyDecoding actor we will regroup SliceData, macroblock_layer, mb_pred, sub_mb_pred, residual and residual_block [7]. The Figure 13 shows how

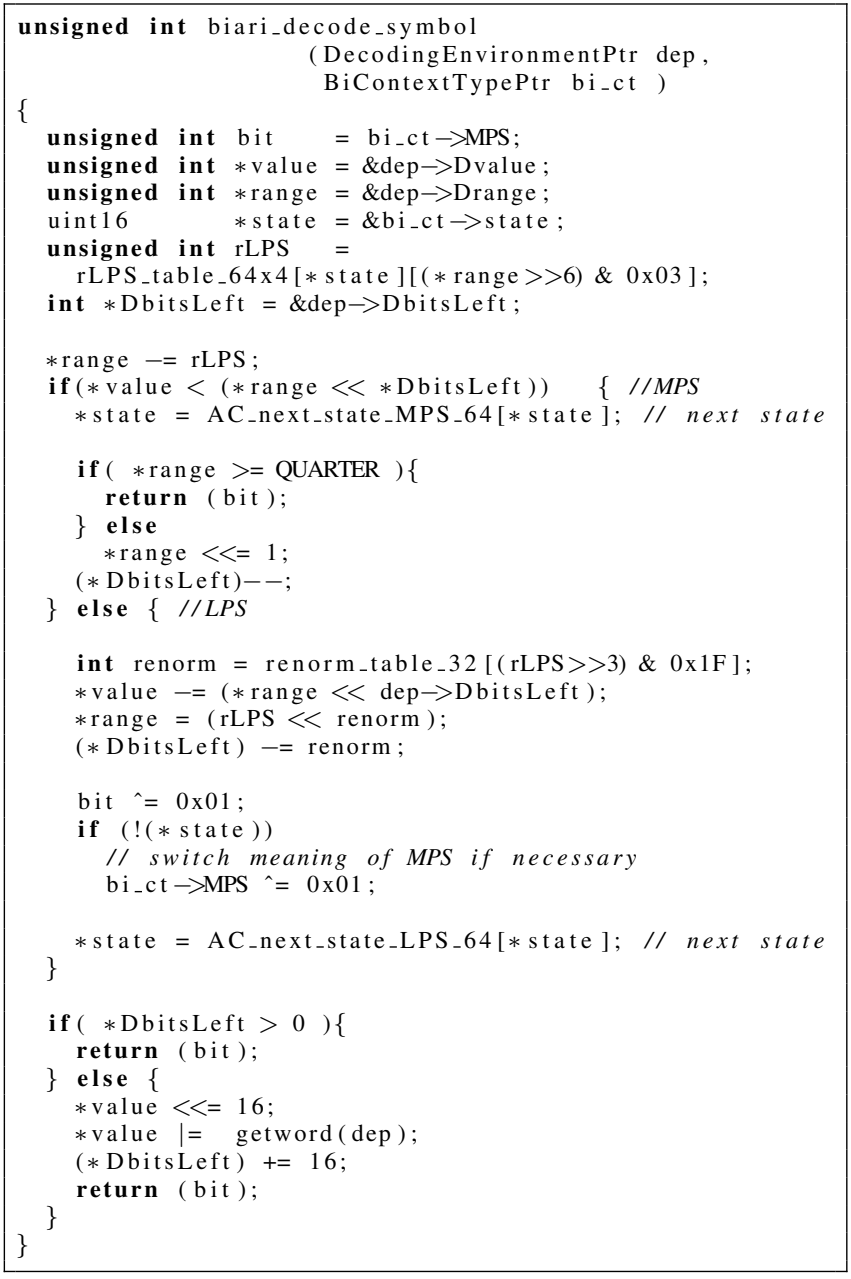

\section{Figure 12. Decoding Decision action written in $\mathrm{C}$ from the $\mathrm{JM}$.}

the sequential AVC/H.264 parser can be transformed to a dataflow model using RVC-CAL.

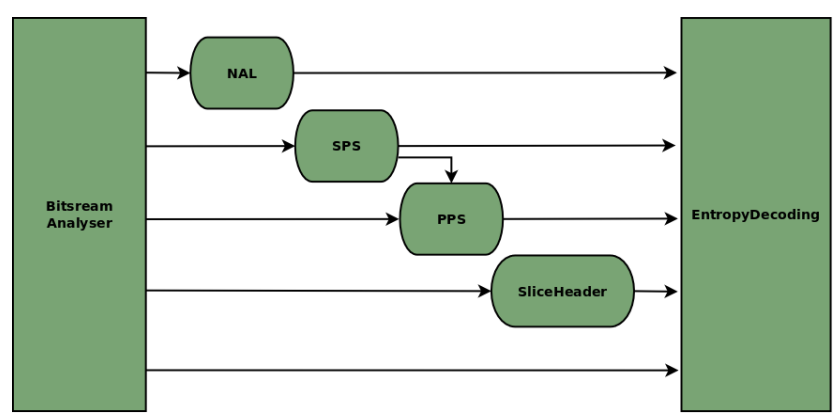

Figure 13. A DataFlow model for the H.264/AVC Parser 


\section{Conclusions}

Implementing the basic $\mathrm{CABAC}$ process in $\mathrm{RVC}-\mathrm{CAL}$ took one month and two months for making it possible to decode the I,P and B slices. Our implementation is supporting all the CABAC encoded Syntax Elements found in the AVC/H.264 standard except the Macroblock field flag. The software generated decoder by our RVC-CAL abstract description can successfully decode a big part of the AVC/H.264 conformance sequences. Using RVC-CAL to interpret the H.264/AVC standard is really easy and this cuts a lot the conception time and actual work compared to other languages. With RVC-CAL is not only possible to implement a type of algorithm in one way but you can implement it as sequential monolithic process, a monolithic dataflow model and to a networked data flow model. Thus implementing the AVC/H.264 Parser in a data flow model and it's entropy decoding engines (CAVLC and CABAC) will achieve higher performances than the actual monolithic data flow model.

\section{References}

[1] S. S. Bhattacharyya, J. Eker, J. W. Janneck, C. Lucarz, M. Mattavelli, and M. Raulet. Overview of the MPEG Reconfigurable Video Coding Framework. Springer journal of Signal Processing Systems. Special Issue on Reconfigurable Video Coding, 2009.

[2] H. Eeckhaut, M. Christiaens, D. Stroobandt, and V. Nollet. Optimizing the critical loop in the H.264/AVC CABAC decoder. Field Programmable Technology, 2006. FPT 2006. IEEE International Conference, 2006.

[3] J. Eker and J. Janneck. CAL Language Report. Technical Report ERL Technical Memo UCB/ERL M03/48, University of California at Berkeley, Dec. 2003.

[4] J. Gorin, M. Raulet, Y. Cheng, H. Lin, N. Siret, K. Sugimoto, and G. Lee. An RVC Dataflow Description of the AVC Constrained Baseline Profile Decoder. In Proceedings of ICIP'09, Nov. 2009.

[5] ISO/IEC FDIS 23001-4. MPEG systems technologies - Part 4: Codec Configuration Representation, 2009.

[6] J. W. Janneck, M. Mattavelli, M. Raulet, and M. Wipliez. Reconfigurable video coding: a stream programming approach to the specification of new video coding standards. In MMSys '10: Proceedings of the first annual ACM SIGMM conference on Multimedia systems, pages 223-234, New York, NY, USA, 2010. ACM.

[7] I. JTC1/SC29/WG11 and I.-T. S. Q.6. Joint Draft ITU-T Rec. H.264 - ISO/IEC 14496-10 / Amd.3 Scalable video coding, 2007.

[8] M. Wipliez, G. Roquier, and J. Nezan. Software Code Generation for the RVC-CAL Language. Journal of Signal Processing Systems, 2009. 